\title{
Are the effects of internal focus instructions different from external focus instructions given during balance training in stroke patients? A double-blind randomised controlled trial
}

\author{
E.C. Kal, MSc ${ }^{\mathrm{a}, \mathrm{b}, \mathrm{c} * \#}$, H. Houdijk, PhD ${ }^{\mathrm{a}, \mathrm{b}}$, J. van der Kamp, PhD ${ }^{\mathrm{b}}$, M.J. Verhoef ${ }^{\text {, }}$ \\ $\mathrm{MSc}^{\mathrm{a}}$, R.B. Prosée, MSc, MD ${ }^{\mathrm{a}}$, E. Groet, $\mathrm{MSc}^{\mathrm{a}}$, M. Winters, $\mathrm{PhD}^{\mathrm{d}}$, C.A.M. van \\ Bennekom, PhD ${ }^{\mathrm{a}, \mathrm{e}}$, E.J.A. Scherder, $\mathrm{PhD}^{\mathrm{c}}$
}

a. Heliomare Rehabilitation Centre, Research \& Development, Wijk aan Zee, The Netherlands

b. Department of Human Movement Sciences, Faculty of Behavioural and Movement Sciences, Vrije Universiteit Amsterdam, The Netherlands

c. Department of Clinical Neuropsychology, Faculty of Behavioural and Movement Sciences, Vrije Universiteit Amsterdam, The Netherlands

d. Research Unit of General Practice in Aalborg, Department of Clinical Medicine, Aalborg University, Aalborg, Denmark

e. Coronel Institute for Occupational and Environmental Health, Academic Medical Centre, Amsterdam, The Netherlands

\footnotetext{
* Corresponding author
}

Email: e.kal@heliomare.nl (EK)

Telephone: +31889208013

† Manon Verhoef sadly passed away in January 2018 


\section{Abstract}

Objective: This study aimed to assess if external focus instructions result in greater improvements in motor skill and automaticity compared to internal focus instructions in stroke patients.

Design: Double-blind randomised controlled trial.

Setting: Inpatient stroke rehabilitation unit.

Subjects: Sixty-three stroke patients $\left(\right.$ Mean $_{\text {age }}=59.6 \pm 10.7$ years; Mean $_{\text {days since stroke }}=$ 28.5 $\pm 16.6 ;$ Median Functional Ambulation Categories $=4)$.

Interventions: Patients were randomly assigned to an internal $(\mathrm{N}=31)$ or external $(\mathrm{N}=32)$ focus instruction group. Both groups practiced a balance board stabilization task, three times per week, for three weeks. Balance performance was assessed at baseline, and after one and three weeks of practice.

Main measures: Primary outcome was the threshold stiffness (Nm/radian) at which patients could stay balanced. Secondary outcomes were patient's sway (root-mean-square error in degrees) at the baseline threshold stiffness under single- and dual-task conditions, and their performance on the Timed-up-and-Go and Utrecht Scale for Evaluation of Rehabilitation. Results: Both groups achieved similar improvements in threshold stiffness ( $\Delta=27.1 \pm 21.1$ $\mathrm{Nm} /$ radian$)$, and single- $\left(\Delta=1.8 \pm 2.3^{\circ}\right.$ root-mean-square error $)$ and dual-task sway $\left(\Delta=1.7 \pm 2.1^{\circ}\right.$ root-mean-square error) after three weeks of practice. No differences were found in improvements in clinical tests of balance and mobility. Patients with comparatively good balance and sensory function, and low attention capacity showed greatest improvements with external focus instructions.

Conclusions: External focus instructions did not result in greater improvement in balance skill in stroke patients compared to internal focus instructions. Results suggest that tailoring instructions to the individual stroke patient may result in optimal improvements in motor skill. 
Key Words: Stroke; Randomised Controlled Trial; External Focus of Attention;

Rehabilitation; Motor Skill; Learning 


\section{Introduction}

Reacquiring motor skills is a challenging and protracted process for patients after stroke. Many patients suffer from cognitive and language deficits. ${ }^{1-3}$ It is therefore pertinent that a therapist uses instructions that are concise, easy to process, but still sufficiently meaningful to help the patient improve motor performance. Recent studies in healthy adults suggest that this may be best achieved with instructions that direct attention 'externally', toward the desired movement outcome. External focus instructions are presumably less cognitively demanding than 'internal' focus instructions, which direct attention toward movement execution itself. Consequently, external focus instructions have been found to result in superior ${ }^{4-9}$ and more automatic $^{10-12}$ motor skill in healthy adults and elderly.

Physical therapists increasingly use external focus strategies when treating stroke patients. ${ }^{13}$ However, it is unknown if external focus instructions are effective for enhancing motor skill acquisition in this patient population. To date, the few studies available have solely investigated the immediate effects of attentional focus on motor performance, and with mixed results. ${ }^{14-17}$ Only one randomised controlled trial has studied the effects of a 4-week intervention on arm function in chronic stroke patients, but it did not find any differences between groups. ${ }^{18}$

Individual patient characteristics may be important to consider when deciding on how to instruct patients. This is particularly true for therapists working in rehabilitation, given the large heterogeneity in the clinical presentation of stroke. This is supported by studies suggesting that external focus instructions could be especially effective for patients with good motor and sensory functioning, poor cognitive capacities, and weak conscious control preferences. $^{13,16,17}$ 
Our aim was to conduct a double-blind randomised controlled trial to assess if external focus instructions result in greater improvements in motor skill and automaticity compared to internal focus instructions in patients after stroke. We hypothesized that patients who practice with external focus instructions would achieve greater improvements in motor skill and automaticity compared to patients who practice with internal focus instructions.

In addition, we explored whether specific patient characteristics influenced the relative efficacy of internal and external instructions. We hypothesized that external instructions would be more efficacious compared to internal instructions for patients with high motor and sensory functioning, low cognitive capacity, and weak conscious control inclination. 


\section{Methods}

\section{Protocol registration}

The study protocol of this double-blind randomised controlled trial was approved by the medical ethical committee of the VU Medical Center in Amsterdam (ID: 2015.354) and preregistered in the Dutch CCMO-register (NL54560.029.15).

\section{Setting, participant recruitment and selection}

Patients with stroke who were receiving inpatient care in rehabilitation centre Heliomare in Wijk aan Zee, The Netherlands were recruited between March 2016 and February 2017. At admission, the rehabilitation physician informed possibly eligible stroke patients about the study in writing and verbally, and invited them to participate. Patients were deemed possibly eligible if they had some degree of walking ability, and seemed able to follow instructions. More specifically, patients were recruited if they suffered a first-ever or recurrent stroke $<6$ months ago, had a Functional Ambulation Categories score $>2$, were able to stand independently $>1$ minute, were able to understand instructions and cooperate with neuropsychological assessment, had no other central nervous system or orthopedic impairments, and had no uncorrected visual or hearing impairment. Patients who were not able to follow instructions, or were not functionally ambulant (Functional Ambulation Categories score $\leq 2$ ) at admission, were monitored throughout their stay. When they achieved Functional Ambulation Categories scores $>2$, their eligibility was further assessed. All patients provided written informed consent prior to inclusion.

\section{Materials and measures}

After inclusion, the following demographic information was collected: General characteristics (age, sex, body weight and height), stroke characteristics (recurrent stroke (yes/no), days since 
stroke and since admission to rehabilitation centre, stroke aetiology and subtype), ${ }^{19}$ general functioning (Utrecht Scale for Evaluation of Rehabilitation), ${ }^{20}$ co-morbidities using the Charlson Cormorbidity Index,${ }^{21}$ motor functioning (Functional Ambulation Categories, ${ }^{22}$ Berg Balance Scale, ${ }^{23}$ Ten Meter Walk-Test; ${ }^{24}$ Timed-up-and-Go), ${ }^{25}$ cognition (education, ${ }^{26}$ attention (D2-attention test), ${ }^{27}$ working memory (Digit-Symbol Substitution Test), ${ }^{28}$ executive functioning (Color-Trails), ${ }^{29}$ presence of aphasia/neglect), sensory functioning (Revised Nottingham Sensory Assessment - lower extremities), ${ }^{30}$ the degree to which patients use conscious control of movement in daily life (Movement-Specific Reinvestment Scale), ${ }^{31}$ and additional hours per week of physical-, occupational-, and sports-therapy received during the intervention period.

A custom-made, validated balance board task ${ }^{32}$ was used to test patients' balance performance, and also for the interventions (Figure 1). This balance board task taxes mediolateral balance control, which is often impaired after stroke. ${ }^{33-35}$ Patients' goal is to stand as still as possible on the balance board, for 30 seconds and without touching the handrail surrounding the board. Task difficulty can be manipulated by adjustment of the board's rotational stiffness $(0-220 \mathrm{Nm} / \mathrm{rad})$. All patients wore a harness to ensure their safety.

\section{$* * *$ Figure 1 here $* * *$}

We used a modified staircase procedure ${ }^{36,37}$ to determine the threshold stiffness $(\mathrm{Nm} / \mathrm{rad})$ at which patients were just able to maintain balance - i.e., keep board deviations below $2.5^{\circ}$ for $70 \%$ of the trial. With this procedure, task difficulty is adjusted on a trial-to-trial basis, based on pre-specified criteria. Please see Brouwer et al. for the full test protocol. ${ }^{32}$ Patients only received unfocused instructions ("stand as still as possible"). Lower threshold stiffness values indicate better balance performance. The rotational stiffness assessment has excellent test- 
retest reliability $(\mathrm{ICC}=0.87)$ and construct validity $(r=-0.56$ with Berg Balance Scale), and a minimum detectable change of $3.20 \mathrm{Nm} / \mathrm{rad}$ on group level. ${ }^{32}$

Next, we measured patients' sway at their baseline threshold stiffness in single-task (as performance measure) and dual-task conditions (as automaticity measure). Sway was defined as the root-mean-square error deviation around the board's average position (degrees). Lower values indicate less sway and, hence, better performance. Patients performed 2 single- and 2 dual-task trials, in the following order: single-task—dual-task—dual-task—single-task. The dual-task was a tone-counting task. ${ }^{11,38}$ Low $(400 \mathrm{~Hz})$ and high $(1000 \mathrm{~Hz})$ tones were presented randomly at 1.5-second intervals in a 1:2 ratio. Patients had to respond as quickly and accurately as possible by saying "yes" whenever a high tone was played, and reported the number of high tones after each 30 -s trial. ${ }^{17}$ Patients performed two single-task tone-counting trials to determine baseline single-task performance. After the balance board measurements, patients' movement-related knowledge was assessed. They verbally described all rules and strategies they had used to perform the balance task. This assessment serves as an extra check to determine the degree to which patients' balance performance relied on conscious motor control; a larger number of rules indicates greater reliance on conscious control. ${ }^{11,39}$

To evaluate the clinically relevant benefits of the interventions, we additionally assessed patients' scores on the Timed-up-and-Go ${ }^{25}$ in single- and dual-task (tone-counting) conditions and on the Utrecht Scale for Evaluation of Rehabilitation-mobility subscale. ${ }^{20}$

\section{Randomization and blinding}

Baseline measurements were performed before randomisation took place. Hence, baseline assessors were blinded to group allocation. Patients were randomly allocated to the external or internal group by an independent researcher (MW) at a remote site who was blinded to the patient at randomisation, except for the variables for which stratification was performed. The 
researcher was otherwise not involved in the trial, nor in patient care. The primary investigator (EK) notified the independent researcher when a new patient had completed the baseline assessment. The independent researcher then used random number generator software (https://www.random.org) to block-randomise patients to the internal or external group (blocks of 4, allocation ratio 1:1; both only known to the independent researcher). Patients were stratified according to lesion location (sub- vs. supratentatorial) and baseline threshold stiffness ( $>60 \mathrm{vs} .<60 \mathrm{Nm} / \mathrm{rad}$ ). Group allocation was shared with the investigator (EK) who provided the intervention but not with the patient or outcome assessors (MV, RP), to minimize the risk of performance, detection and attrition bias.

\section{Interventions}

Patients in both groups practiced the balance board task for three weeks, three times per week, with 15 single-task trials per session. In the first practice session, the baseline threshold stiffness was used in the first block of five trials. Depending on patients' average performance (Table 1), stiffness was either increased $(+20 \% \mathrm{Nm} / \mathrm{rad})$, maintained, or decreased $(-20 \%$ $\mathrm{Nm} / \mathrm{rad}$ ) in the next block, to ensure that task difficulty remained challenging throughout practice. Before each trial, the external focus group was instructed to "focus on the board, and keep the board as still as possible", while the internal group was instructed to "focus on your feet, and keep your feet as still as possible". 9,12

\section{*** Table 1 here ***}

After each session, we checked adherence. Patients rated (1) the effort needed to focus as instructed, (2) the effort needed to maintain the instructed focus throughout the trial, and (3) the effectiveness of the instructed focus, by putting a cross on a horizontal $10 \mathrm{~cm}$-line $(0 \mathrm{~cm}=$ "very little/effective"; $10 \mathrm{~cm}=$ "very much/completely ineffective"). ${ }^{40}$ Scores below $5.0 \mathrm{~cm}$ indicate 
that patients were able to adhere to instructions, and found these to be more helpful than harmful for their performance.

\section{Outcome assessments}

Blinded assessors (MV, RP) performed outcome assessments after one and three weeks of practice. Both followed an identical procedure as the baseline assessment, except that the Timed-up-and-Go and Utrecht Scale for Evaluation of Rehabilitation were re-assessed after 3 weeks only. Patients were explicitly instructed not to tell which instructions they had received during practice.

The primary outcome measure was patients' individual threshold rotational stiffness. Secondary outcome measures were patients' sway at their baseline threshold stiffness in single-task and dual-task conditions, and their scores on the Timed-up-and-Go and Utrecht Scale for Evaluation of Rehabilitation.

\section{Data processing}

Potentiometer data and verbal responses on the tone-counting task were sampled at $1000 \mathrm{~Hz}$ using LabVIEW (National Instruments; Austin; Texas), and analysed with Matlab (Mathworks, Natick MA, USA). The balance board's potentiometer data was filtered with a bidirectional, low-pass $(8 \mathrm{~Hz})$ Butterworth filter. We used non-linear regression to determine the patients' individual threshold stiffness (see Brouwer et al. for details). ${ }^{32}$ To determine single- and dual-task sway, we calculated the root-mean-square error of board deviations (in degrees) per trial. For the tone-counting task, we calculated reaction time (ms), and response and counting accuracy (\%) per trial. These were collapsed in a composite score (Equation 1). ${ }^{41}$ Tone-counting dual-task performance was operationalized by calculating dual-task costs (DTCs; Equation 2). ${ }^{42}$ Positive DTC indicates performance deterioration in dual-task versus single-task conditions. 


$$
\begin{gathered}
\text { Tone Counting Performance }=\frac{\text { Mean Accuracy }}{\text { Reaction Time }} \times 100 \%[1] \\
\text { DTC }=\frac{\text { Single Task }- \text { Dual Task }}{\text { Single Task }} \times 100 \%[2]
\end{gathered}
$$

Patient's self-reported verbal rules were transcribed verbatim and scored offline (EK) - only movement-related rules were scored. If conditions were measured twice (sway, tonecounting), values were averaged.

\section{Sample size calculations \& Statistics}

Power analysis (G* $\mathrm{G}^{*}$ power) showed a sample size of 52 to be sufficient to detect a small-tomoderate effect $(f=.20)$, based on a repeated measures ANOVA (within-between interaction), alpha of .05, beta of .80, 2 groups, and $r$ of 0.5 . Expecting a drop-out of $10-15 \%, 60$ patients (30/group) were needed.

All data were analysed with SPSS version 20.0. Patient characteristics were described with their appropriate central estimate and measures of dispersion, and were compared between groups to check whether randomization was successful.

Generalized Estimating Equations (GEEs) were used to compare learning effectiveness between groups. We used an autoregressive correlation matrix to define this dependency. First, we used GEE to model the association between the primary outcome, threshold stiffness, and the predictors group (external vs. internal), time (Baseline, 1 week, 3 weeks), and their interaction. Learning differences were considered present in case of significant group by time interaction. Similar GEEs were used for the analysis of the secondary outcomes, single- and dual-task sway. We a-priori decided to add the covariate "handrail support" to both sway analyses, as this factor likely influences sway. Similarly, tone-counting dual-task costs served as covariate in the dual-task sway analysis, to correct for any task- 
prioritization differences. Finally, we conducted GEEs (predictors group, time(baseline -3 weeks follow-up), interaction) on Utrecht Scale for Evaluation of Rehabilitation-mobility subscale and single- and dual-task Timed-up-and-Go. Again, tone-counting dual-task costs were added to the dual-task analysis. For all GEE-analyses, Holm-Bonferroni t-tests followed up significant effects. ${ }^{43}$ For these post-hoc t-tests, we presented the adjusted mean differences between groups or test sessions. Cohen's $d$ served as measure of effect size.

We performed per-protocol analyses, and additional intention-to-treat analyses to determine whether attrition influenced results. For intention-to-treat, missing cases were imputed based on the overall median improvement in the respective outcome measures. ${ }^{44} \mathrm{We}$ assumed that drop-outs would show similar improvements as the other patients. Therefore, we estimated the median percentage improvement per outcome measure, and used these to estimate patients' performance on the missing test sessions.

We a-priori decided to investigate whether cognition (Color-Trails, Digit-Symbol Substitution Test, D2-attention test), motor capacity (Berg Balance Scale), conscious control inclination (Movement-Specific Reinvestment Scale), and sensory functioning (Revised Nottingham Sensory Assessment - lower extremities) had a different effect on learning in the external group than in the internal group. Variables were submitted to the respective GEE-models of stiffness, single- and dual-task sway in turn. Variables were labeled 'effect modifiers' when the group $\mathrm{x}$ time $\mathrm{x}$ 'variable' term was significant. To assess how an effect modifier influenced learning per group, separate linear regression analyses were run with absolute learning improvements (3 weeks - Baseline) as dependent variable. Effect modification analyses were restricted to per-protocol analyses of the full three-week learning period. 


\section{Results}

Sixty-three patients were included. Figure 2 shows the flow of the study. A total of 51 patients completed the whole intervention and assessment after 3 weeks.

\section{$* * *$ Figure 2 here $* * *$}

Table 2 lists baseline characteristics of all included patients. There were no apparent baseline group differences, except that the external group seemed to be heavier than the internal group. Weight was positively associated with threshold stiffness at all three test sessions $(B$ 's $\geq 0.523$, $p$ 's $\leq 0.011)$. Therefore, it was included as covariate in the analysis of threshold stiffness. Both groups indicated that they focused their attention as instructed during practice, confirming that they adhered to the assigned intervention. Please see appendix 1 for more details.

\section{$* * *$ Table 2 here $* * *$}

\section{Primary outcome}

Table 3A summarizes threshold rotational stiffness per measurement session. Appendix 2 shows the development of rotational stiffness throughout practice.

After three weeks of practice, the external group had improved by $25.8 \pm 18.6 \mathrm{Nm} / \mathrm{rad}$, while the internal group had improved by $28.4 \pm 23.2 \mathrm{Nm} / \mathrm{rad}$ (main effect of time, $p<0.001$; Table 4A). However, this improvement in rotational stiffness did not differ between groups ( $p=0.653$ ). Overall, post-hoc tests showed that patients significantly improved over the whole learning period $(\Delta=27.1 \pm 20.9 \mathrm{Nm} / \mathrm{rad}, p<0.001)$, between baseline and 1 week of practice $(\Delta=19.1 \pm 17.6 \mathrm{Nm} / \mathrm{rad}, p<0.001)$, and between 1 week and 3 weeks of practice $(\Delta=8.0 \pm 10.5 \mathrm{Nm} / \mathrm{rad}, p<0.001)$. Appendix 3 lists details of all post-hoc tests. Intention-to-treat analysis yielded similar results (Table 4A), suggesting that attrition did not influence results. 


\section{Secondary outcomes}

Table 3A summarizes the sway (root-mean-square error degrees) in single- and dual-task conditions per group, while Table 3B presents the results of the Timed-up-and-Go test and the mobility subscale of the Utrecht Scale for Evaluation of Rehabilitation.

Regarding single-task sway, both the external $\left(\Delta=0.93 \pm 1.97^{\circ}\right)$ and internal group $\left(\Delta=1.37 \pm 2.37^{\circ}\right)$ showed substantial improvements after three weeks of practice (main effect of time, $p<0.001$; Table 4A). However, results also showed that the external group showed larger initial improvements than the internal group (significant group by time interaction, $p=0.031)$. Specifically, post-hoc tests showed that the external group significantly improved between baseline and 1 week of practice $\left(\Delta=0.97 \pm 1.72^{\circ}, p=0.016\right)$, but did not further improve afterwards $\left(\Delta=-0.05 \pm 0.84^{\circ}, p=0.779\right)$. The internal group showed the opposite pattern. It did not significantly improve in the first week $\left(\Delta=0.60 \pm 2.14^{\circ}, p=0.320\right)$, but only achieved significant improvements between 1 and 3 weeks of practice $\left(\Delta=0.77 \pm 1.29^{\circ}, p=0.018\right.$; Appendix 3). Results were similar with intention-to-treat analyses (Table 4A).

With regard to dual-task sway, both the external $\left(\Delta=1.28 \pm 1.77^{\circ}\right)$ and internal group $\left(\Delta=0.69 \pm 1.66^{\circ}\right)$ showed improvements after three weeks of practice (main effect of time, $p<0.001$; Table 4A). However, this improvement in dual-task sway did not differ between groups ( $p=0.330$; Table 4A). Overall, post-hoc tests showed that patients significantly improved over the whole learning period $\left(\Delta=0.98 \pm 1.88^{\circ}, p<0.001\right)$, and showed nearsignificant improvements between baseline and 1 week of practice $\left(\Delta=0.62 \pm 2.03^{\circ}, p=0.060\right)$, and between 1 week and 3 weeks of practice $\left(\Delta=0.36 \pm 1.43^{\circ} ; p=0.076\right)$. Intention-to-treat analysis yielded similar results (Table 4A). 
With regard to the clinical tests of general balance and mobility, after three weeks of practice the external and internal group both showed significant improvements in single-task $\left(\Delta_{\text {external }}=5.55 \pm 6.07\right.$ seconds $; \Delta_{\text {internal }}=5.95 \pm 6.60$ seconds $)$ and in dual-task $\left(\Delta_{\text {external }}=5.78 \pm 7.99\right.$ seconds; $\Delta_{\text {internal }}=6.27 \pm 7.43$ seconds) Timed-up-and-Go performance. They also both showed significant improvements $\left(\Delta_{\text {external }}=10.2 \pm 6.0\right.$ points; $\Delta_{\text {internal }}=7.2 \pm 6.2$ points $)$ in the mobility subscale of the Utrecht Scale for Evaluation of Rehabilitation (main effects of time, $p$ 's $<0.001$; Table 4B). For all three outcomes, these improvements did not differ between groups ( $p \geq 0.094)$. Intention-to-treat analyses yielded similar results for all three outcomes (Table 4B).

\section{$* * *$ Tables $3 \& 4$ here $* * *$}

\section{Influence of patient characteristics on effectiveness of focus instructions}

We found that patients with comparatively good balance and sensory functioning, and with low attentional capacity generally showed stronger improvements in balance board performance with external than with internal instructions.

First, baseline Berg Balance Scale score predicted whether patients improved their threshold rotational stiffness more with external or with internal focus instructions (Wald $\chi^{2}=29.64$, $p<0.001)$. In the internal group, worse Berg Balance Scale scores were predictive of greater improvements in threshold stiffness $(B=-1.665)$. This pattern was less pronounced for the external group $(B=-0.392)$.

Second, sensory functioning of the lower extremities (Revised Nottingham Sensory Assessment) modified learning on all three balance board outcomes (threshold rotational stiffness: Wald $\chi^{2}=17.69, p=0.001$; single-task sway: Wald $\chi^{2}=21.59, p<0.001$; dual-task sway: Wald $\chi^{2}=6.709, p=0.082$ ). In the external group, better sensory functioning predicted greater improvement in threshold stiffness $(B=0.485)$ and single-task sway $(B=0.152)$. In contrast, in 
the internal group lower sensory functioning predicted greater improvement in threshold stiffness $(B=-1.410)$ and single-task sway $(B=-0.061)$. Effects on dual-task sway were similar but less distinct.

Finally, attention (D2-attention test) scores predicted whether dual-task sway improved most with external or internal focus instructions (Wald $\chi^{2}=7.843, p=0.049$ ). In the external group, lower attention scores predicted greater improvement in dual-task sway $(B=-0.013)$. In the internal group, by contrast, better attention scores predicted greater improvement in dual-task sway $(B=0.008)$. 


\section{Discussion}

This RCT found that the external group did not show greater improvements in the primary outcome, threshold rotational stiffness, compared to the internal focus group. Analysis of the secondary outcome measure of single-task sway revealed that the external group showed greater improvements early in learning after 1 week, but not after 3 weeks of practice. Yet, the external group did not show enhanced automaticity: Both groups showed comparable improvements in dual-task sway. In line with this, both groups reported a similar amount of declarative movement-related knowledge (appendix 1), which also indicates that balance performance was similarly automated. ${ }^{11,39}$ Finally, the lack of group differences in the Timedup-and-Go and Utrecht Scale for Evaluation of Rehabilitation suggests that both attentional focus interventions had similar clinical benefits. Overall, external focus instructions did not result in greater improvements in motor skill and automaticity compared to internal focus instructions in rehabilitating stroke patients.

Our results are different from those of the majority of studies in healthy adults, which reported that external focus interventions result in superior motor skill and dual-task performance. ${ }^{4-12}$ One explanation for this stems from the single-task sway analysis. This suggested that external instructions may accelerate learning in the very short term - within the first week of practice - but not in the longer term - after three weeks of practice. Notably, in healthy adults, balance board studies that reported greater improvements in performance with an external focus typically concerned practice periods of a few days. ${ }^{6,9,12}$ Possibly, benefits in healthy adults will also decrease or even disappear with prolonged practice.

From a clinical viewpoint, one could speculate that accelerated learning with external focus instructions may increase patients' feelings of competence, ${ }^{45}$ motivation, and self-efficacy, and could eventually shorten inpatient rehabilitation duration. Note, though, that accelerated 
learning was not observed in stiffness and automaticity, and clinical benefits were similar for both groups. Also, patients found it more difficult to use external focus instructions, which possibly decreases motivation. This difficulty with focusing externally may be related to patients' overall strong inclination to consciously control their movements, which is evidenced by patients' high scores on the Movement-Specific Reinvestment Scale (Table 2). $31,46,47$

The effect modification analyses partly confirmed our hypothesis that the effects of focus instructions would be dependent on patients' motor functioning, sensory functioning, cognition, and conscious control inclination. Specifically, external instructions resulted in greater improvements in balance board performance for patients with comparatively good balance and sensory functioning, while internal instructions were more effective for patients with larger impairments. This skill-dependent effect of attentional focus was also found in a previous study that compared the immediate effects in stroke patients. ${ }^{17}$ Wulf et al. ${ }^{12}$ argued that an internal focus hinders learning because it disrupts automaticity. Our findings suggest that this is only the case if some degree of sensory function and motor skill has been established in the first place (cf. Masters and Maxwell ${ }^{48}$ ). In addition, when it comes to improving dual-tasking, we found that patients with more severe attentional deficits benefitted more from external focus instructions than from internal focus instructions. This is in line with the idea that an internal focus is more attention-demanding than an external focus. ${ }^{10,12}$ Focusing internally would therefore be more easy for patients with intact attentional capacity, especially in dual-task situations when resources need to be shared with an additional cognitive task.

For rehabilitation practice, these results imply that a tailored use of attentional focus instructions may be more effective than an exclusive reliance on external focus instructions. This study suggests that a patients' motor, sensory, and attentional functioning may be 
important. However, we do not know how therapists should weigh these different characteristics; e.g. what to do if a patient has both good balance and large attentional capacity? A challenge for future research is to replicate our analyses, investigate other possibly relevant factors such as imagery capacity, ${ }^{49}$ and explore how different factors interact.

To the best of our knowledge, this is the first RCT that compared attentional focus instructions on motor skill and automaticity in rehabilitating stroke patients. Our results seem generalizable to the larger stroke population, given the large heterogeneity in terms of patient characteristics. Recent reviews have emphasized the need for motor learning research to improve on reporting, methodology, sample size, and statistics. ${ }^{50-52}$ Accordingly, we preregistered the study design, a-priori defined the primary and secondary outcomes, and blinded outcome assessment and group allocation. Further, this study involved a comparatively large number of patients and an adequately long practice period. Finally, intention-to-treat analyses confirmed the robustness of our results to drop-outs and missing cases.

A limitation is the absence of a control group that received no specific instructions, making it impossible to assess whether the focus instructions hindered or promoted learning. Also, we only used one specific standardised focus instruction per group. These exact same instructions have been used extensively in prior research in healthy adults and elderly, ${ }^{9,12}$ larger contrast between the interventions might have been achieved by using a larger set of attentional focus instructions. Another limitation was that it is impossible to blind the person providing the intervention to group allocation. A third point concerns the clinical relevance of the chosen tasks. While often used for research purposes, ${ }^{6,9,12}$ the balance task primarily taxes mediolateral balance control in a laboratory setting. Future studies may compare the effects of focus instructions on walking, or more complex (e.g., perturbation) and functional balance tasks. ${ }^{53}$ Fourth, we did not include a retention test after a couple of weeks or months, and thus 
could not compare the longer-term retention of skill improvements. A final issue concerns our effect modification analyses. In contrast to the factors planned for this analysis, the sensory functioning test (Revised Nottingham Sensory Assessment) was added to our analysis plan when the study was already underway. During a meeting on a related topic, a physical therapist argued that patients may compensate for impaired sensory functioning with conscious, internally focused control. Although our data seem to confirm this hypothesis, further research is needed to replicate these findings. This is especially true given that our sample size was powered for the analysis of our primary outcome variable only

\section{Conclusions}

No overall benefit was found of external focus instructions over internal focus instructions for improving balance skill and automaticity after stroke. For clinical practice, our results suggest that it may be more effective to tailor instructions to the individual patient, rather than uniformly use external instructions for all patients. 


\section{Clinical Messages}

- External focus instructions did not enhance learning on a balance task in rehabilitating stroke patients compared to internal focus instructions

- External focus instructions seem more effective than internal focus instructions for patients with higher balance (Berg Balance Scale) and sensory (Nottingham Sensory Assessment) capacity, and with lower attention capacity (D2-attention test)

\section{Acknowledgements}

The authors thank Chantal Nieuwenhuizen, Rafaël Brouwer, and Rosalie Denneman for their contribution to data collection and pilot measurements. We also would like to thank Roelof van Ouwerkerk, Bas Vermeeren, Thijs Zwinnen, and Micha Paalman (department of Physics and Medical Technology, VU Medical Centre, Amsterdam, The Netherlands) and Bert Clairbois and Siro Otten (Department of Human Movement Sciences, VU University, Amsterdam, The Netherlands) for their contributions to the development of the balance board and software.

\section{Funding}

This project was funded by the Revalidatiefonds (number: R2015042;

www.revalidatiefonds.nl). The funding party had no involvement in data collection, analysis, or interpretation. It was also not involved in writing the paper or in the decision to submit it for publication.

\section{Conflict of interest statement}

The authors declare no conflicts of interest.

\section{Data availability}

All underlying research materials (including anonymized data, customized software, SPSS- 
files and syntaxes) can be accessed by contacting the primary investigator (e.kal@heliomare.nl). 


\section{References}

1. Nys GMS, van Zandvoort MJE, de Kort PLM, Jansen BPW, de Haan EHF, Kappelle LJ. Cognitive disorders in acute stroke: Prevalence and clinical determinants. Cerebrovasc Dis. 2007;23(5-6):408-416. doi:10.1159/000101464.

2. Zinn S, Bosworth HB, Hoenig HM, Swartzwelder HS. Executive function deficits in acute stroke. Arch Phys Med Rehabil. 2007;88(2):173-180. doi:10.1016/j.apmr.2006.11.015.

3. Dickey L, Kagan A, Lindsay MP, Fang J, Rowland A, Black S. Incidence and profile of inpatient stroke-induced aphasia in Ontario, Canada. Arch Phys Med Rehabil. 2010;91(2):196-202. doi:10.1016/j.apmr.2009.09.020.

4. Wulf G. Attentional focus and motor learning: a review of 15 years. Int Rev Sport Exerc Psychol. 2013;6(1):77-104. doi:10.1080/1750984X.2012.723728.

5. Lohse KR, Sherwood DE, Healy AF. How changing the focus of attention affects performance, kinematics, and electromyography in dart throwing. Hum Mov Sci. 2010;29(4):542-555. doi:10.1016/j.humov.2010.05.001.

6. Shea $\mathrm{CH}$, Wulf G. Enhancing motor learning through external-focus instructions and feedback. Hum Mov Sci. 1999;18(4):553-571. doi:10.1016/S0167-9457(99)00031-7.

7. Wulf G, Dufek JS, Lozano L, Pettigrew C. Increased jump height and reduced EMG activity with an external focus. Hum Mov Sci. 2010;29(3):440-448.

doi:10.1016/j.humov.2009.11.008.

8. Zachry T, Wulf G, Mercer J, Bezodis N. Increased movement accuracy and reduced EMG activity as the result of adopting an external focus of attention. Brain Res Bull. 2005;67(4):304-309. doi:10.1016/j.brainresbull.2005.06.035.

9. Chiviacowsky S, Wulf G, Wally R. An external focus of attention enhances balance learning in older adults. Gait Posture. 2010;32(4):572-575.

doi:10.1016/j.gaitpost.2010.08.004.

10. Kal EC, van der Kamp J, Houdijk H. External attentional focus enhances movement automatization: A comprehensive test of the constrained action hypothesis. Hum Mov Sci. 2013. doi:10.1016/j.humov.2013.04.001.

11. Poolton JM, Maxwell JP, Masters RSW, Raab M. Benefits of an external focus of attention: Common coding or conscious processing? J Sports Sci. 2006;24(1):89-99.

12. Wulf G, McNevin N, Shea $\mathrm{CH}$. The automaticity of complex motor skill learning as a function of attentional focus. Q J Exp Psychol A. 2001;54(4):1143-1154. doi:10.1080/0272498014300011.

13. Kal E, van den Brink H, Houdijk H, et al. How physical therapists instruct patients with stroke: an observational study on attentional focus during gait rehabilitation after stroke. Disabil Rehabil. 2018;40(10):1154-1165.

doi:10.1080/09638288.2017.1290697.

14. Fasoli SE, Trombly CA, Tickle-Degnen L, Verfaellie MH. Effect of instructions on functional reach in persons with and without cerebrovascular accident. Am J Occup Ther. 2002;56(4):380-390. doi:10.5014/ajot.56.4.380.

15. Muckel S, Mehrholz J. Immediate effects of two attention strategies on trunk control on patients after stroke. A randomized controlled pilot trial. Clin Rehabil. 2014;28(7):632636. doi:10.1177/0269215513513963.

16. Durham K, Sackley CM, Wright CC, Wing AM, Edwards MG, Van Vliet P. Attentional focus of feedback for improving performance of reach-to-grasp after stroke: a randomised crossover study. Physiotherapy. 2014;10:108-115.

17. Kal EC, Van Der Kamp J, Houdijk H, Groet E, Van Bennekom CAM, Scherder EJA. Stay focused! The effects of internal and external focus of attention on movement 
automaticity in patients with stroke. PLoS One. 2015.

doi:10.1371/journal.pone.0136917.

18. Kim GJ, Hinojosa J, Rao AK, Batavia M, O’Dell MW. A randomized trial on the effects of attentional focus on motor training of the upper extremity using robotics with individuals after chronic stroke. Arch Phys Med Rehabil. 2017;98(10):1924-1931. doi:10.1016/j.apmr.2017.06.005

19. Bamford J, Sandercock P, Dennis M, Warlow C, Burn J. Classification and natural history of clinically identifiable subtypes of cerebral infarction. Lancet. 1991;337(8756):1521-1526. doi:10.1016/0140-6736(91)93206-O.

20. Post MW, van de Port IG, Kap B, Berdenis van Berlekom SH. Development and validation of the Utrecht Scale for Evaluation of Clinical Rehabilitation (USER). Clin Rehabil. 2009;23:909-917. doi:10.1177/0269215509341524.

21. Charlson M, Szatrowski TP, Peterson J, Gold J. Validation of a combined comorbidity index. J Clin Epidemiol. 1994;47(11):1245-1251. doi:10.1016/0895-4356(94)90129-5.

22. Holden MK, Gill KM, Magliozzi MR, Nathan J, Piehl-Baker L. Clinical gait assessment in the neurologically impaired. Reliability and meaningfulness. Phys Ther. 1984;64(1):35-40.

23. Berg KO, Wood-Dauphinee SL, Williams JI, Maki B. Measuring balance in the elderly: Validation of an instrument. Can J Public Health.1992; S7-11.

24. Flansbjer UB, Holmbäck AM, Downham D, Patten C, Lexell J. Reliability of gait performance tests in men and women with hemiparesis after stroke. J Rehabil Med. 2005;37(2):75-82. doi:10.1080/16501970410017215.

25. Podsiadlo D, Richardson S. The timed "Up and Go": A test of basic functional mobility for frail elderly persons. J Am Geriatr Soc. 1991;39(2):142-148. doi:10.1016/j.ctim.2008.10.005.

26. Verhage F. Intelligentie en leeftijd: onderzoek bij Nederlanders van twaalf tot zevenzeventig jaar [Intelligence and Age: study with Dutch people aged 12 to 77]. Assen Van Gorcum. 1964.

27. Brinckenkamp R, Oosterveld P. De d2 Aandachts- En Concentratietest, Nederlandse Bewerking van de "Aufmerksamheits Belastung Test" van R. Brinckenkamp. [The D2 attention and concentration test, Dutch version of the "Aufmerksamheits Belastung Test" of R. Brinckenkamp] Amsterdam: Hogrefe Publishing; 2007.

28. Wechsler D. Wechsler Memory Scale - Revised. New York, NY, USA: The Psychological Corporation; 1987.

29. D'Elia LF, Satz P, Uchiyama CL, White T. Color Trails Test: Professional Manual. FL: Odessa; 1996.

30. Stolk-Hornsveld F, Crow JL, Hendriks EP, van der Baan R, Harmeling-van der Wel BC. The Erasmus MC modifications to the (revised) Nottingham Sensory Assessment: a reliable somatosensory assessment measure for patients with intracranial disorders. Clin Rehabil. 2006;20(2):160-172. doi:10.1191/0269215506cr932oa.

31. Kal E, Houdijk H, van der Wurff P, et al. The inclination for conscious motor control after stroke: validating the Movement-Specific Reinvestment Scale for use in inpatient stroke patients. Disabil Rehabil. 2016. doi:10.3109/09638288.2015.1091858.

32. Brouwer R, Kal E, Van der Kamp J, Houdijk H. Validation of the stabilometer balance test: Bridging the gap between clinical and research based balance control assessments for stroke patients. Gait Posture. Under review. Available as thesis from: https://dspace.library.uu.nl/handle/1874/351824.

33. Mercer VS, Freburger JK, Chang S-H, Purser JL. Measurement of paretic-lowerextremity loading and weight transfer after stroke. Phys Ther. 2009;89(7):653-664. doi:10.2522/ptj.20080230. 
34. Cheng PT, Liaw MY, Wong MK, Tang FT, Lee MY, Lin PS. The sit-to-stand movement in stroke patients and its correlation with falling. Arch Phys Med Rehabil. 1998;79(9):1043-1046. doi:10.1016/S0003-9993(98)90168-X.

35. Cheng PT, Wu SH, Liaw MY, Wong AMK, Tang FT. Symmetrical body-weight distribution training in stroke patients and its effect on fall prevention. Arch Phys Med Rehabil. 2001;82(12):1650-1654. doi:10.1053/apmr.2001.26256.

36. Karmali F, Chaudhuri SE, Yi Y, Merfeld DM. Determining thresholds using adaptive procedures and psychometric fits: evaluating efficiency using theory, simulations, and human experiments. Exp Brain Res. 2016;234(3):773-789. doi:10.1007/s00221-0154501-8.

37. Taylor MM, Creelman CD. PEST: Efficient Estimates on Probability Functions. $J$ Acoust Soc Am. 1967;41(4):782-787. doi:10.1121/1.1910407.

38. Poolton JM, Masters RSW, Maxwell JP. The relationship between initial errorless learning conditions and subsequent performance. Hum Mov Sci. 2005;24(3):362-378. doi:10.1016/j.humov.2005.06.006.

39. Kleynen M, Braun SM, Bleijlevens MH, et al. Using a Delphi technique to seek consensus regarding definitions, descriptions and classification of terms related to implicit and explicit forms of motor learning. PLoS One. 2014;9(6):e100227. doi:10.1371/journal.pone.0100227.

40. Van Ginneken WF, Poolton JM, Masters RSW, Capio CM, Kal EC, van der Kamp J. Comparing the effects of conscious monitoring and conscious control on motor performance. Psychol Sport Exerc. 2017;30:145-152.

doi:10.1016/j.psychsport.2017.03.001.

41. Smulders K, van Swigchem R, de Swart BJM, Geurts ACH, Weerdesteyn V. Community-dwelling people with chronic stroke need disproportionate attention while walking and negotiating obstacles. Gait Posture. 2012;36(1):127-132. doi:10.1016/j.gaitpost.2012.02.002.

42. McCulloch K. Attention and dual-task conditions: Physical therapy implications for individuals with acquired brain injury. J Neurol Phys Ther. 2007;31(3):104-118.

43. Holm S. A simple sequentially rejective multiple test procedure. Scand J Stat. 1979;6:65-70. doi:10.2307/4615733.

44. White IR, Horton NJ, Carpenter J, Pocock SJ. Strategy for intention to treat analysis in randomised trials with missing outcome data. BMJ. 2011;342:d40.

45. Deci EL, Ryan RM. Self-determination theory: A macrotheory of human motivation, development, and health. Can Psychol. 2008;49(3):182-185. doi:10.1037/a0012801.

46. Orrell AJ, Masters RSW, Eves FF. Reinvestment and movement disruption following stroke. Neurorehabil Neural Repair. 2009;23(2):177-183. doi:10.1177/1545968308317752.

47. Kleynen M, Braun SM, Beurskens AJ, Verbunt JA, de Bie RA, Masters RSW. Investigating the Dutch Movement-Specific Reinvestment Scale in people with stroke. Clin Rehabil. 2012;27(2):160-165. doi:10.1177/0269215512448381.

48. Masters R, Maxwell J. The theory of reinvestment. Int Rev Sport Exerc Psychol. 2008;1(2):160-183. doi:10.1080/17509840802287218.

49. Sakurada T, Nakajima T, Morita M, Hirai M, Watanabe E. Improved motor performance in patients with acute stroke using the optimal individual attentional strategy. Sci Rep. 2017;7. doi:10.1038/srep40592.

50. Lohse K, Buchanan T, Miller M. Underpowered and overworked: Problems with data analysis in motor learning studies. J Mot Learn Dev. 2016;4(1):37-58. doi:10.1123/jmld.2015-0010.

51. Kal E, Winters M, van der Kamp J, et al. Is implicit motor learning preserved after 
stroke? A systematic review with meta-analysis. PLoS One. 2016.

doi:10.1371/journal.pone.0166376.

52. Kal EC, Prosée RB, Winters M, van der Kamp J. Does implicit motor learning lead to faster automatization of motor skills compared to explicit motor learning? A systematic review. PLoS One. 2018. doi:10.1371/journal.pone.0203591.

53. de Melker Worms JLA, Stins JF, van Wegen EEH, Loram ID, Beek PJ. Influence of focus of attention, reinvestment and fall history on elderly gait stability. Physiol Rep. 2017;5(1):e13061. doi:10.14814/phy2.13061. 


\section{TABLES}

Table 1. Criteria for evaluating success during practice sessions.

\begin{tabular}{|c|c|c|}
\hline \multicolumn{3}{|c|}{ Performance criteria for practice sessions } \\
\hline $\begin{array}{c}\text { Average \% of trial } \\
\text { duration that board } \\
\text { deviates }<2.5 \text { degrees }\end{array}$ & $\begin{array}{c}\text { Number of trials } \\
\text { participant grabbed } \\
\text { handrail for support }\end{array}$ & $\begin{array}{c}\text { Stiffness for } \\
\text { next block of } \\
5 \text { trials }\end{array}$ \\
\hline$>70 \%$ & 1 or 2 trials & Stiffness-20\% \\
\hline$>70 \%$ & $>2$ trials & No change \\
\hline $60 \%-70 \%$ & 1 or 2 trials & No change \\
\hline $60 \%-70 \%$ & $>2$ trials & Stiffness $+20 \%$ \\
\hline$<60 \%$ & Any number & Stiffness $+20 \%$ \\
\hline
\end{tabular}

NB: Handrail support was scored by observation by the experimenter. 
Table 2. Baseline characteristics per group.

\begin{tabular}{|c|c|c|}
\hline Variable & $\begin{array}{l}\text { Internal Focus } \\
\text { Group }(\mathbf{N}=\mathbf{3 1})\end{array}$ & $\begin{array}{c}\text { External Focus } \\
\text { Group }(\mathbf{N}=32)\end{array}$ \\
\hline \multicolumn{3}{|l|}{ General characteristics } \\
\hline Age in years (mean \pm SD) & $58.5 \pm 10.3$ & $60.7 \pm 11.1$ \\
\hline Sex (male/female) & $23 / 8$ & $20 / 12$ \\
\hline Weight in $\mathrm{kg}($ mean \pm SD) & $77.6 \pm 12.1$ & $83.7 \pm 16.1$ \\
\hline Height in $\mathrm{cm}($ mean \pm SD) & $176.8 \pm 8.7$ & $176.0 \pm 9.0$ \\
\hline Leg length in $\mathrm{cm}(\text { mean } \pm \mathrm{SD})^{\mathrm{c}}$ & $102.9 \pm 6.5$ & $103.5 \pm 5.8$ \\
\hline \multicolumn{3}{|l|}{ Stroke characteristics } \\
\hline Days since stroke at baseline $^{\mathrm{a}}($ mean $\pm \mathrm{SD})$ & $30.5 \pm 21.3$ & $26.6 \pm 10.3$ \\
\hline Days since admission at baseline (mean \pm SD) & $14.3 \pm 10.9$ & $11.7 \pm 8.4$ \\
\hline Stroke aetiology (haemorrhagic/infarction) & $7 / 24$ & $8 / 24$ \\
\hline \multicolumn{3}{|l|}{ Stroke subtype } \\
\hline Total Anterior Circulation Stroke & 2 & 1 \\
\hline Partial Anterior Circulation Stroke & 15 & 15 \\
\hline Lacunar Stroke & 8 & 9 \\
\hline Posterior Circulation Stroke & 6 & 7 \\
\hline Recurrent Stroke (yes/no) & $3 / 28$ & $4 / 28$ \\
\hline $\begin{array}{l}\text { Revised Nottingham Sensory Assessment - lower } \\
\text { extremities }(0-80 ; \text { mean } \pm \mathrm{SD})^{\mathrm{a}}\end{array}$ & $71.7 \pm 10.5$ & $74.8 \pm 5.2$ \\
\hline Charlson Comorbidity Index $\left(\right.$ mean \pm SD) ${ }^{\mathrm{a}}$ & $0.52 \pm 0.6$ & $0.78 \pm 1.2$ \\
\hline Additional hours of therapy/week $\left(\right.$ mean \pm SD) ${ }^{\mathrm{a}}$ & $8.1 \pm 2.3$ & $8.3 \pm 1.7$ \\
\hline \multicolumn{3}{|l|}{ Motor characteristics } \\
\hline Berg Balance Scale $(0-56 \text {; mean } \pm \text { SD })^{\mathrm{a}, \mathrm{b}}$ & $46.3 \pm 10.1$ & $49.0 \pm 7.3$ \\
\hline Functional Ambulation Categories $(0-5 ;$ median \pm IQR) & $4 \pm 2$ & $4 \pm 1$ \\
\hline Ten Meter Walk Test $(\mathrm{s} ; \text { mean } \pm \mathrm{SD})^{\mathrm{a}}$ & $17.1 \pm 9.9$ & $14.1 \pm 8.6$ \\
\hline Timed-up-and-Go -ST (s; mean \pm SD) ${ }^{\mathrm{a}}$ & $19.5 \pm 11.1$ & $16.6 \pm 11.3$ \\
\hline Timed-up-and-Go -DT (s; mean \pm SD) ${ }^{\mathrm{a}}$ & $21.1 \pm 11.7$ & $18.0 \pm 11.8$ \\
\hline USER-MOB $(0-35 ; \text { mean+SD })^{\mathrm{b}}$ & $23.3 \pm 6.7$ & $24.4 \pm 7.0$ \\
\hline \multicolumn{3}{|l|}{ Cognitive characteristics } \\
\hline Education level (1-7; median \pm IQR) & $5 \pm 2$ & $5 \pm 2$ \\
\hline USER-COG $(0-50 ; \text { mean+SD) })^{\mathrm{a}, \mathrm{b}}$ & $43.5 \pm 4.5$ & $44.5 \pm 5.7$ \\
\hline Aphasia (yes/no) & $7 / 24$ & $3 / 29$ \\
\hline Neglect (yes/no) & $9 / 22$ & $6 / 26$ \\
\hline Attention (D2-attention test; mean \pm SD) ${ }^{\mathrm{d}}$ & $118.6 \pm 48.4$ & $120.6 \pm 41.4$ \\
\hline Working Memory (DSST; mean \pm SD) ${ }^{c}$ & $43.5 \pm 16.9$ & $47.1 \pm 18.3$ \\
\hline Executive Function (Color Trails Test; mean \pm SD) ${ }^{\mathrm{e}}$ & $0.91 \pm 0.56$ & $0.97 \pm 0.47$ \\
\hline \multicolumn{3}{|l|}{ Conscious motor control preference } \\
\hline MSRS-Total (mean \pm SD) & $37.7 \pm 10.5$ & $34.0 \pm 11.0$ \\
\hline MSRS-CMP (mean \pm SD) & $22.6 \pm 6.2$ & $20.3 \pm 6.1$ \\
\hline MSRS-MS-C (mean \pm SD) & $15.2 \pm 5.4$ & $13.7 \pm 5.9$ \\
\hline
\end{tabular}

NB: The group comparisons were performed to check whether randomization was successful. Near-significant differences are emphasized. ${ }^{\text {a }}$ Variable was not normally distributed, therefore a Mann-Whitney U test was performed; ${ }^{\mathrm{b}}$ Data unavailable for 1 patient; ${ }^{\mathrm{c}}$ Data unavailable for 4 patients; ${ }^{\mathrm{d}}$ Data unavailable for 5 patients; ${ }^{\mathrm{e}}$ Data unavailable for 7 patients. Abbreviations: $\mathrm{CMP}=\mathrm{Conscious}$ Motor Processing subscale; $\mathrm{COG}=\mathrm{Cognitive}$ subscale; CTT=Color Trails Test; DSST=Digit Symbol Substitution Test; IQR=Interquartile range; MOB=Mobility subscale; MS-C=Movement Self-Consciousness subscale; MSRS=Movement-Specific Reinvestment Scale; $\mathrm{s}=$ seconds; $\mathrm{SD}=$ Standard deviation; $\mathrm{SE}=$ Standard error; USER=Utrecht Scale for Evaluation of Rehabilitation; 
Table 3. Summary of balance board (A) and clinical test (B) results (mean \pm standard error). Data presented here concern the raw unadjusted data for patients for whom complete data was available (i.e., per protocol; $\mathrm{N}=51$ ).

\begin{tabular}{|c|c|c|c|}
\hline \multicolumn{4}{|c|}{ A. Balance Board Measures } \\
\hline Threshold Stiffness & Test Session & Internal Focus & External Focus \\
\hline \multirow{3}{*}{$\begin{array}{l}\text { Threshold Rotational Stiffness } \\
\text { (Newton meter/radian) }\end{array}$} & Baseline & $44.08 \pm 7.13$ & $40.00 \pm 5.05$ \\
\hline & 1 week & $25.03 \pm 5.01$ & $20.89 \pm 3.89$ \\
\hline & 3 weeks & $15.64 \pm 4.46$ & $14.21 \pm 3.45$ \\
\hline \multicolumn{4}{|l|}{ Single-Task Sway } \\
\hline \multirow[t]{3}{*}{ Single-Task sway (degrees RMSE) } & Baseline & $2.48 \pm 0.51$ & $2.34 \pm 0.36$ \\
\hline & 1 week & $1.46 \pm 0.41$ & $0.78 \pm 0.16$ \\
\hline & 3 weeks & $0.57 \pm 0.17$ & $0.66 \pm 0.20$ \\
\hline \multirow{3}{*}{$\begin{array}{l}\text { Single-Task Handrail Support } \\
\text { (number of times) }\end{array}$} & Baseline & $1.56 \pm 0.33$ & $1.77 \pm 0.30$ \\
\hline & 1 week & $0.70 \pm 0.25$ & $0.58 \pm 0.21$ \\
\hline & 3 weeks & $0.46 \pm 0.17$ & $0.23 \pm 0.09$ \\
\hline \multicolumn{4}{|l|}{ Dual-Task Sway } \\
\hline \multirow[t]{3}{*}{ Dual-Task sway (degrees RMSE) } & Baseline & $2.13 \pm 0.48$ & $2.58 \pm 0.42$ \\
\hline & 1 week & $1.30 \pm 0.39$ & $1.15 \pm 0.33$ \\
\hline & 3 weeks & $0.69 \pm 0.19$ & $0.72 \pm 0.19$ \\
\hline \multirow{3}{*}{$\begin{array}{l}\text { Dual-Task Handrail Support } \\
\text { (number of times) }\end{array}$} & Baseline & $1.35 \pm 0.26$ & $2.08 \pm 0.44$ \\
\hline & 1 week & $0.98 \pm 0.37$ & $0.67 \pm 0.20$ \\
\hline & 3 weeks & $0.50 \pm 0.24$ & $0.60 \pm 0.21$ \\
\hline \multirow[t]{3}{*}{ Tone-counting dual-task costs $(\%)$} & Baseline $^{b}$ & $9.15 \pm 3.78$ & $4.63 \pm 3.45$ \\
\hline & 1 week & $5.23 \pm 3.56$ & $3.34 \pm 2.17$ \\
\hline & 3 weeks & $1.14 \pm 3.42$ & $0.48 \pm 2.13$ \\
\hline \multicolumn{4}{|c|}{ B. Clinical Balance \& Mobility Tests } \\
\hline Single-Task Timed-up-and-Go & Test Session & Internal Focus & External Focus \\
\hline \multirow[t]{2}{*}{ Single-Task Timed-up-and-Go(s) } & Baseline & $20.45 \pm 2.48$ & $17.89 \pm 2.37$ \\
\hline & 3 weeks & $14.64 \pm 2.55$ & $12.34 \pm 1.66$ \\
\hline \multicolumn{4}{|l|}{ Dual-Task Timed-up-and-Go ${ }^{\mathbf{a}}$} \\
\hline \multirow[t]{2}{*}{ Dual-Task Timed-up-and-Go (s) } & Baseline & $22.04 \pm 2.60$ & $19.11 \pm 2.48$ \\
\hline & 3 weeks & $16.18 \pm 2.50$ & $12.81 \pm 1.54$ \\
\hline \multirow{2}{*}{$\begin{array}{l}\text { Timed-up-and-Go -tone-counting } \\
\text { dual-task costs }(\%)\end{array}$} & Baseline & $5.18 . \pm 3.41$ & $1.77 \pm 3.56$ \\
\hline & 3 weeks & $7.67 \pm 3.49$ & $-1.08 \pm 4.94$ \\
\hline \multicolumn{4}{|l|}{ USER-Mobility } \\
\hline \multirow[t]{2}{*}{ USER-Mobility } & Baseline $^{c}$ & $23.54 \pm 1.41$ & $23.27 \pm 1.26$ \\
\hline & 3 weeks $^{d}$ & $30.71 \pm 1.05$ & $33.44 \pm 0.35$ \\
\hline
\end{tabular}


NB: ${ }^{\text {a }}$ One internal group member excluded as outlier; ${ }^{\mathrm{b}}$ No data for 1 internal group member due to malfunctioning microphone; ${ }^{\mathrm{c}}$ No data for 1 internal group member; ${ }^{\mathrm{d}}$ No data for 1 external and 3 internal group members; Abbreviations: RMSE=Root-mean-square error; USER=Utrecht Scale for Evaluation of Rehabilitation; 
Table 4. Results of per protocol $(\mathrm{N}=51)$ and intention-to-treat $(\mathrm{N}=63)$ GEE-analyses of balance board (A) and clinical test results (B).

\begin{tabular}{|c|c|c|c|c|}
\hline A. Balance Board Measures & \multicolumn{2}{|c|}{ Per protocol $(\mathrm{N}=51)$} & \multicolumn{2}{|c|}{ Intention-to-treat $(\mathrm{N}=63)$} \\
\hline Threshold Stiffness & Wald $\chi^{2}$ & $p$ & Wald $\chi^{2}$ & $p$ \\
\hline Group (Internal, External) & 1.47 & 0.226 & 2.594 & 0.107 \\
\hline Time (Baseline, 1 week, 3 weeks) & 85.82 & $<0.001$ & 116.73 & $<0.001$ \\
\hline Group x Time & 0.85 & 0.653 & 1.04 & 0.595 \\
\hline Weight $^{\mathrm{a}}$ & 9.64 & 0.002 & 20.52 & $<\mathbf{0 . 0 0 1}$ \\
\hline \multicolumn{5}{|l|}{ Single-Task Sway } \\
\hline Group (Internal, External) & 0.40 & 0.526 & 0.00 & 0.952 \\
\hline Time (Baseline, 1 week, 3 weeks) & 15.46 & $<0.001$ & 23.29 & $<0.001$ \\
\hline Group x Time & 6.92 & 0.031 & 6.40 & 0.041 \\
\hline Handrail Support & 11.57 & $<0.001$ & 14.01 & $<0.001$ \\
\hline \multicolumn{5}{|l|}{ Dual-Task Sway $^{b}$} \\
\hline Group (Internal, External) & 0.27 & 0.603 & 0.71 & 0.400 \\
\hline Time (Baseline, 1 week, 3 weeks) & 14.33 & 0.001 & 25.06 & $<\mathbf{0 . 0 0 1}$ \\
\hline Group x Time & 2.22 & 0.330 & 2.76 & 0.252 \\
\hline Handrail Support & 4.97 & 0.026 & 6.89 & 0.009 \\
\hline Tone-counting dual-task costs & 6.47 & 0.011 & 3.92 & 0.048 \\
\hline $\begin{array}{c}\text { B. Clinical Balance \& Mobility } \\
\text { Tests }\end{array}$ & \multicolumn{2}{|c|}{ Per protocol $(\mathrm{N}=51)$} & \multicolumn{2}{|c|}{ Intention-to-treat $(\mathrm{N}=63)$} \\
\hline Single-Task Timed-up-and-Go & Wald $\chi^{2}$ & $p$ & Wald $\chi^{2}$ & $p$ \\
\hline Group (Internal, External) & 0.65 & 0.421 & 1.16 & 0.282 \\
\hline Time (Baseline, 3 weeks) & 40.96 & $<0.001$ & 51.96 & $<\mathbf{0 . 0 0 1}$ \\
\hline Group x Time & 0.05 & 0.823 & 0.14 & 0.710 \\
\hline \multicolumn{5}{|l|}{ Dual-Task Timed-up-and-Gob } \\
\hline Group (Internal, External) & 0.84 & 0.359 & 1.38 & 0.240 \\
\hline Time (Baseline, 3 weeks) & 35.42 & $<\mathbf{0 . 0 0 1}$ & 45.05 & $<0.001$ \\
\hline Group x Time & 0.00 & 0.970 & 0.01 & 0.907 \\
\hline Tone-counting dual-task costs & 5.11 & 0.024 & 5.44 & 0.020 \\
\hline \multicolumn{5}{|l|}{ USER-Mobility $^{\mathrm{c}}$} \\
\hline Group (Internal, External) & 1.07 & 0.302 & 3.33 & 0.068 \\
\hline Time (Baseline, 3 weeks) & 89.27 & $<0.001$ & 99.44 & $<0.001$ \\
\hline Group x Time & 2.81 & 0.094 & 2.59 & 0.108 \\
\hline
\end{tabular}

NB: aSensitivity analysis revealed the effect of group $(p=0.611)$ and group by time interaction $(p=0.653)$ to be similar when weight was excluded from the stiffness analysis; ${ }^{b}$ One internal group member was excluded as outlier, but sensitivity analyses showed that the group by time interaction remained nonsignificant when this patient was included $(p=0.574)$; ${ }^{\mathrm{c} B a s e l i n e ~ U S E R ~ m o b i l i t y ~ s c o r e s ~ u n a v a i l a b l e ~ f o r ~} 6$ patients.

Abbreviations: RMSE=Root-mean-square error; USER=Utrecht Scale for Evaluation of Rehabilitation; 


\section{FIGURES}

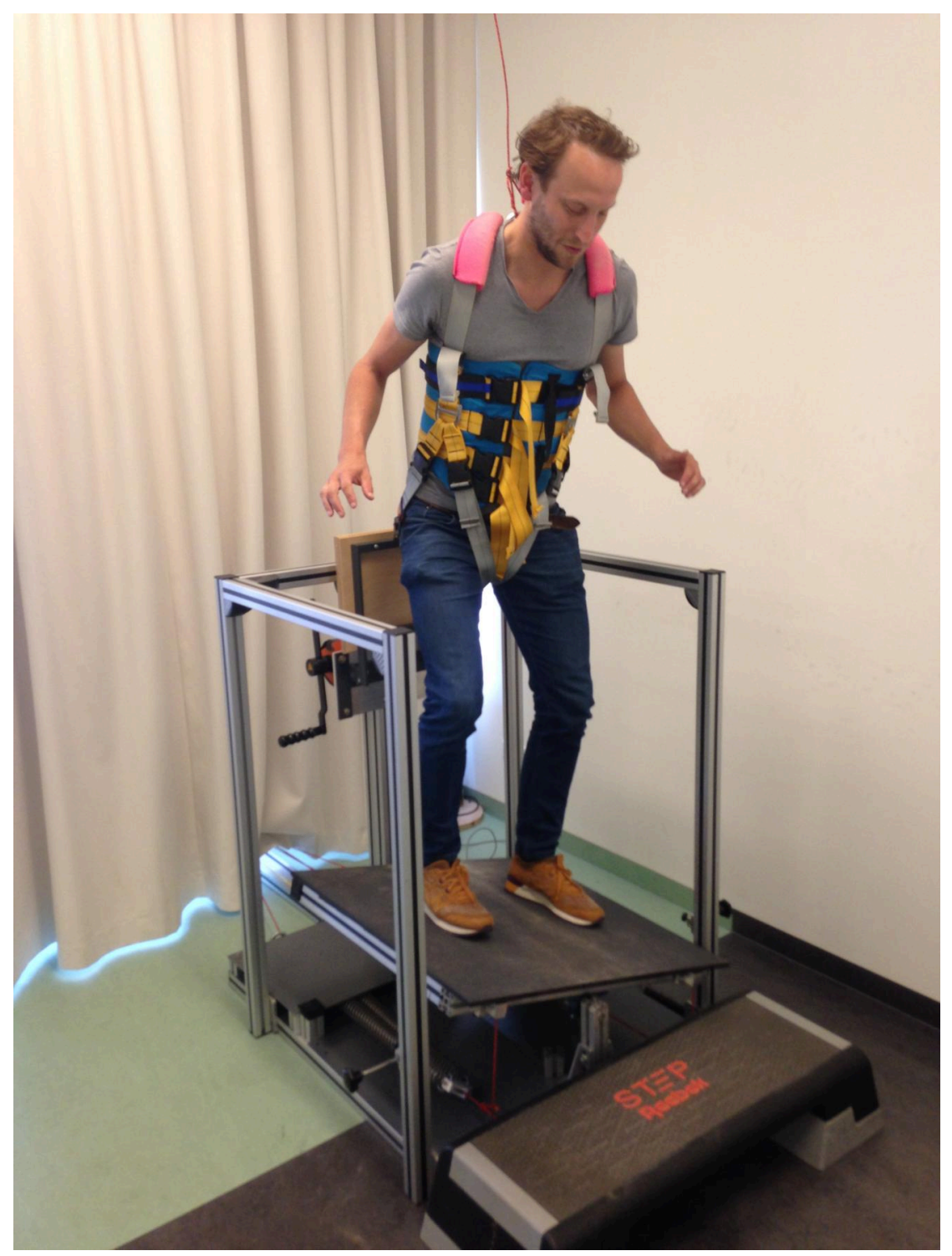

Figure 1. Balance board set-up. Springs were attached to each side of the front of the balance board. Rotational stiffness could be adjusted $(0-220 \mathrm{Nm} / \mathrm{rad})$ by using either one or two parallel springs on each side, by altering the springs' moment arm, or by changing the springs themselves $(800 \mathrm{~N} / \mathrm{m}$ vs. $390 \mathrm{~N} / \mathrm{m})$. Patients wore a safety harness. 


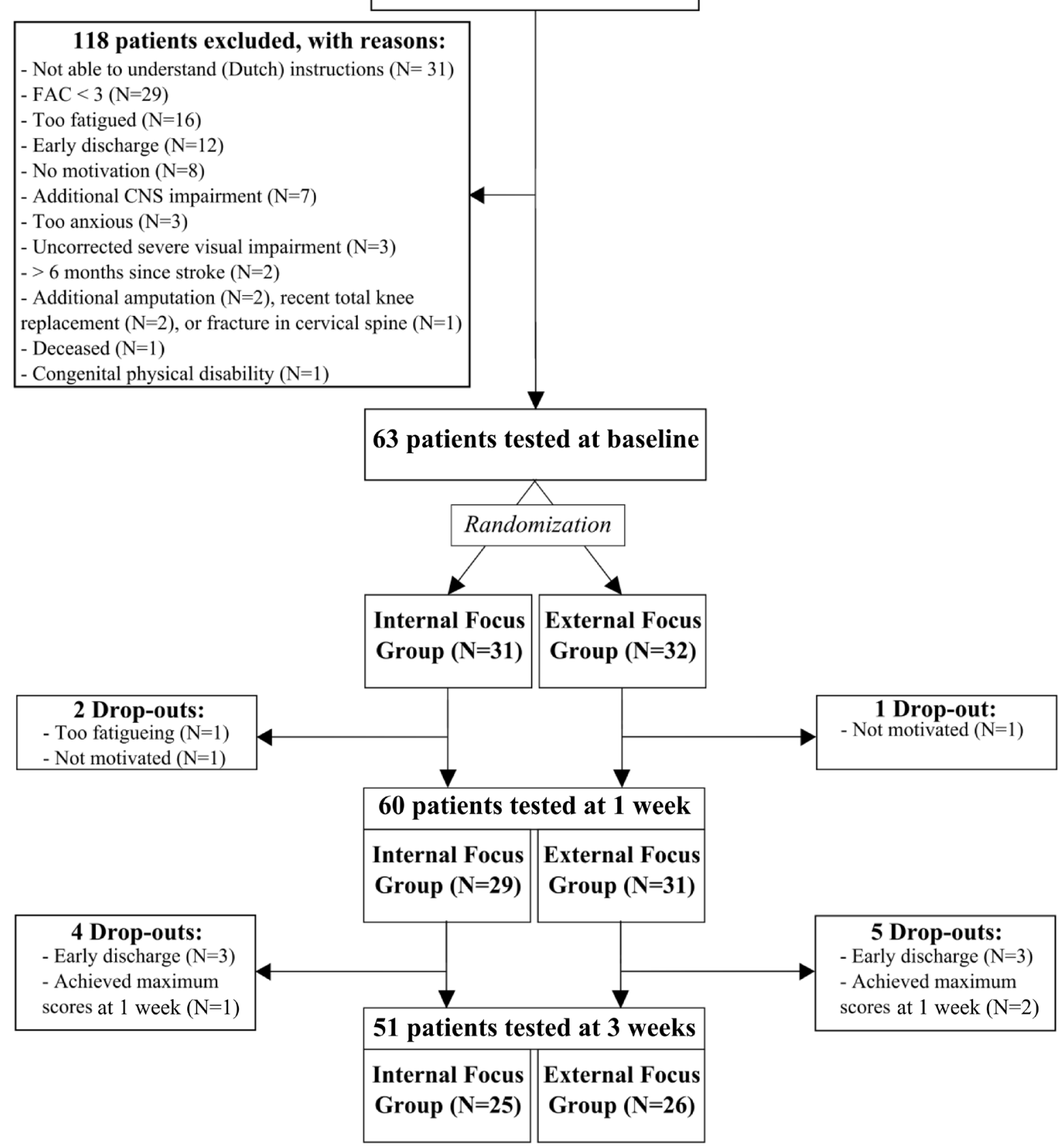

Figure 2. Study flow. Abbreviations: $\mathrm{CNS}=\mathrm{Central}$ nervous system; $\mathrm{FAC}=$ Functional Ambulation Categories; 


\section{Appendix 1. Results of manipulations checks}

Adherence to instructions

Patients were able to maintain their focus throughout the trial, and found the provided instructions effective for improving their performance (average scores on all three checks $<$ $5.0 \mathrm{~cm})$. The external group did tend to rate it more effortful to focus as instructed (mean=3.23, SE $=0.32 ; t(49)=1.860, p=0.070, d=0.260)$ and to maintain this focus throughout each trial (mean=4.08, $\mathrm{SE}=0.31 ; t(49)=1.737, p=0.089, d=0.243)$ compared to the internal group (mean=2.30, $\mathrm{SE}=0.38$; and mean $=3.18, \mathrm{SE}=0.42$; respectively). Both groups judged the effectiveness of instructions similar $\left(\right.$ mean $_{\text {External }}=3.37, \mathrm{SE}=0.33$ vs. mean $_{\text {Internal }}=2.88$, $\mathrm{SE}=0.36$, respectively; $t(49)=1.01, p=0.318, d=0.141)$.

Amount of movement-related declarative knowledge of balance board performance The external and internal group reported a similar number of movement-related rules at baseline (mean=2.42, $\mathrm{SE}=0.24$, vs. mean $=2.04, \mathrm{SE}=0.28$, respectively), after 1 week (mean $=2.19, \mathrm{SE}=0.22$, vs. mean $=1.96, \mathrm{SE}=0.23$, respectively) and after 3 weeks (mean=2.23, $\mathrm{SE}=0.31$, vs. mean=1.84, $\mathrm{SE}=0.28$, respectively; $t$ 's(49) $\leq 1.049, p \geq 0.299, d \leq 0.150)$. The number of movement-related rules did also not change over time in either group ( $t$ 's $(24-$ $\left.25) \leq 0.894, p^{\prime} \mathrm{s} \geq 0.380, d^{\prime} \mathrm{s} \leq 0.179\right)$. 


\section{Appendix 2. Threshold rotational stiffness over time}

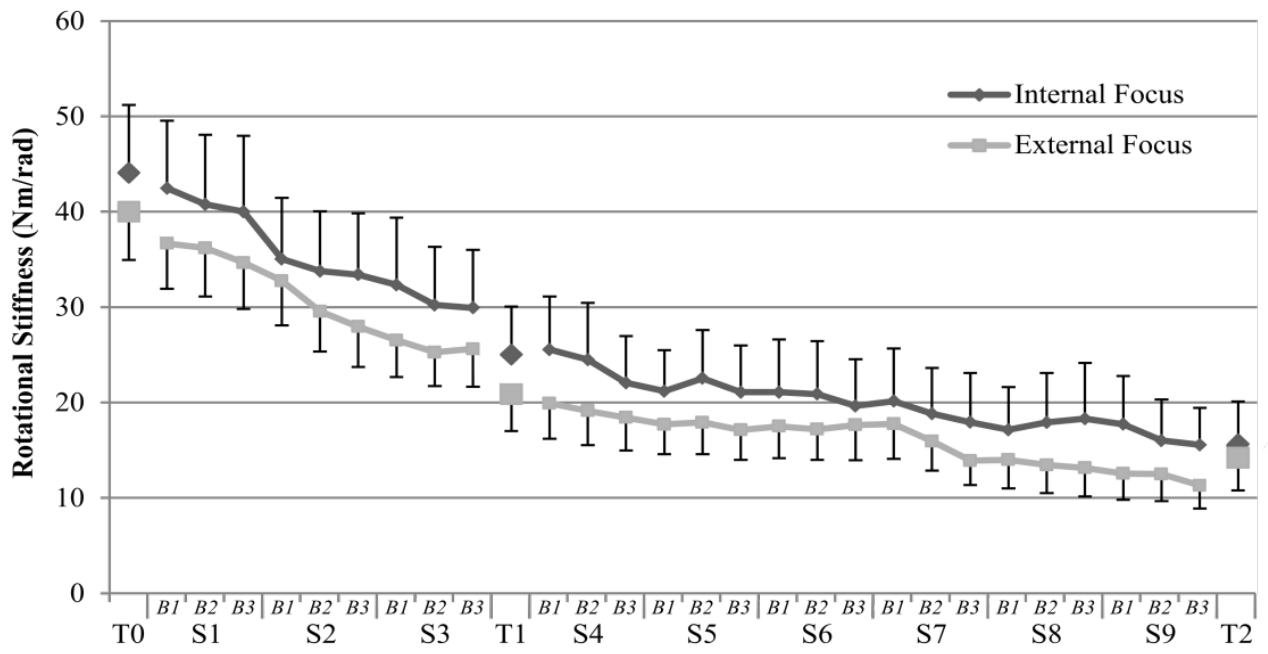

Figure A2. Development of threshold rotational stiffness (Mean Newton meter/radian \pm standard error) over time for all patients who completed the whole 3-week training period (per protocol; $\mathrm{N}=51$ ). Results are presented for both groups for the test sessions at baseline (T0), after 1 week of practice (T1), and after 3 weeks of practice (T2). Results are also presented per block (B1-B3) of each practice session (S1-S9) for both groups. Lower values indicate better performance. 
Appendix 3. Details of post-hoc tests per analysis

\begin{tabular}{|c|c|c|c|}
\hline Analysis & & & \\
\hline $\begin{array}{l}\text { Threshold rotational } \\
\text { stiffness }\end{array}$ & $\begin{array}{l}\text { Both groups } \\
\text { combined }\end{array}$ & External group & Internal group \\
\hline Baseline vs. 1 week & $\begin{array}{c}t(50)=7.735 \\
p<0.001 \\
d=1.083\end{array}$ & N/A & $\mathrm{N} / \mathrm{A}$ \\
\hline 1 week vs. 3 weeks & $\begin{array}{c}t(50)=5.463 \\
p<0.001 \\
d=0.765\end{array}$ & $\mathrm{~N} / \mathrm{A}$ & $\mathrm{N} / \mathrm{A}$ \\
\hline Baseline vs. 3 weeks & $\begin{array}{c}t(50)=9.248 \\
p<0.001 \\
d=1.295\end{array}$ & N/A & N/A \\
\hline Single-task sway & $\begin{array}{c}\text { Both groups } \\
\text { combined }\end{array}$ & External group & Internal group \\
\hline Baseline vs. 1 week & N/A & $\begin{array}{c}t(25)=2.887 \\
p=0.016 \\
d=0.566\end{array}$ & $\begin{array}{c}t(24)=1.405 \\
p=0.320 \\
d=0.281\end{array}$ \\
\hline 1 week vs. 3 weeks & N/A & $\begin{array}{c}t(25)=-0.280 \\
p=0.779 \\
d=0.055\end{array}$ & $\begin{array}{c}t(24)=2.976 \\
p=0.018 \\
d=0.595\end{array}$ \\
\hline Baseline vs. 3 weeks & $\mathrm{N} / \mathrm{A}$ & $\begin{array}{c}t(25)=2.403 \\
p=0.016 \\
d=0.471\end{array}$ & $\begin{array}{c}t(24)=2.898 \\
p=0.020 \\
d=0.580\end{array}$ \\
\hline Dual-task sway & $\begin{array}{c}\text { Both groups } \\
\text { combined }\end{array}$ & External group & Internal group \\
\hline Baseline vs. 1 week & $\begin{array}{c}t_{T 0-T 1}(49)=1.769 \\
p=0.060 \\
d=0.310\end{array}$ & N/A & N/A \\
\hline 1 week vs. 3 weeks & $\begin{array}{c}t_{T 0-T 2}(49)=1.035 \\
p=0.076 \\
d=0.254\end{array}$ & N/A & N/A \\
\hline Baseline vs. 3 weeks & $\begin{array}{c}t(49)=3.698 \\
p<0.001 \\
d=0.528\end{array}$ & $\mathrm{~N} / \mathrm{A}$ & $\mathrm{N} / \mathrm{A}$ \\
\hline
\end{tabular}

NB: N/A = not applicable; 